\title{
Evaluación de la capacidad funcional en escolares con fisiología de corazón univentricular
} Assessment of functional capacity among students with univentricular heart

\author{
Dra. Camila Ampuero ${ }^{a}$, Lic. Richard Silva ${ }^{b}$, Dr. Paulo Valderramac ${ }^{c}$ Lic. Emilio Covarrubias ${ }^{b}$ \\ Dr. Patricio Astudillo ${ }^{a}$, Dra. Pamela Zelada ${ }^{c, d}$ y Dr. Cristian Claveria ${ }^{c}$
}

\section{RESUMEN}

Se realizó un estudio transversal en escolares con corazón univentricular en estadio pos-bypass total de ventrículo derecho con el objetivo de determinar la capacidad funcional basal mediante el test de marcha en 6 minutos e identificar posibles factores determinantes. Participaron 30 pacientes con una mediana de edad de 12 años. Dieciocho pacientes fueron de sexo masculino. La mediana de distancia recorrida fue de 551,3 metros, un $84 \%$ de la distancia teórica para población pediátrica sana. Las variables talla, presión arterial sistólica pretest y saturación arterial de oxígeno de reposo se asociaron significativamente con la distancia recorrida en el modelo de regresión lineal múltiple. No hubo asociación significativa en los metros caminados respecto de las variables sexo, estado nutricional, dignóstico cardiológico inicial, número de cirugías previas y edad al momento del bypass total de ventrículo derecho. Palabras clave: test de la marcha de 6 minutos, rehabilitación cardiaca, procedimiento de Fontan, niño, tolerancia al ejercicio.

http: / / dx.doi.org/10.5546/ aap.2020.343

Texto completo en inglés:

http: / / dx.doi.org/10.5546/ aap.2020.eng.343

Cómo citar: Ampuero C, Silva R, Valderrama P P, Covarrubias E, et al. Evaluación de la capacidad funcional en escolares con fisiología de corazón univentricular. Arch Argent Pediatr 2020;118(5):343-347.

a. División Pediatría, Facultad de Medicina, Pontificia Universidad Católica de Chile.

b. Servicio de Kinesiología, Hospital Clínico Red de Salud UC-CHRISTUS.

c. Departamento de Cardiología y Enfermedades Respiratorias Pediátricas, División Pediatría, Facultad de Medicina, Pontificia Universidad Católica de Chile.

d. Unidad de Cardiología Infantil. Servicio de Pediatría. Complejo Asistencial Dr. Sótero del Río, Santiago, Chile.

Correspondencia:

Dr. Cristian Clavería: claveria@med.puc.cl

Financiamiento: Ninguno.

Conflicto de intereses: Ninguno que declarar.

Recibido: 24-10-2019

Aceptado: $14-5-2020$

\section{INTRODUCCIÓN}

El bypass total del ventrículo derecho (BPTVD) es un procedimiento quirúrgico paliativo realizado en pacientes con corazón univentricular (CUV) que ha permitido un aumento de la sobrevida en esta población. Las ventajas de esta nueva fisiología incluyen la normalización de la saturación arterial de oxígeno $\left(\mathrm{SaO}_{2}\right)$ y la eliminación de la sobrecarga crónica de volumen a expensas de aumentar la presión venosa sistémica y de disminuir el gasto cardíaco en reposo y en ejercicio. ${ }^{1}$

La capacidad funcional (CF) es un predictor independiente de morbimortalidad en los pacientes con cardiopatías congénitas (CC), incluidos aquellos con fisiología de CUV. Su evaluación es importante para obtener información sobre la salud del sistema cardiopulmonar y los factores que pueden afectarla. $^{2}$

El test de marcha en 6 minutos (TM6M) es un método seguro, sencillo y de bajo costo para medir la CF en los niños sanos, con valores estandarizados que permiten realizar estudios comparativos. ${ }^{3}$ Su uso en niños con CC ha sido útil y fiable en la evaluación y seguimiento durante programas de rehabilitación. ${ }^{4}$

Este estudio buscó determinar la CF mediante la aplicación del TM6M en los pacientes con CUV que se encuentran, actualmente, en la etapa pos-BPTVD e identificar sus posibles factores determinantes.

\section{POBLACIÓN Y MÉTODO \\ Pacientes}

Estudio transversal en pacientes de 6 hasta 16 años de edad con diagnóstico de CUV en estadio pos-BPTVD operados en el Hospital Clínico UC-Christus entre los años 2000 y 2013. El muestreo fue por conveniencia basado en la revisión de una base de datos de la misma Institución. Se excluyeron los pacientes usuarios de oxígeno, fármacos beta-bloqueadores, marcapasos y con diagnósticos de arritmias. Además, se excluyeron aquellos con afecciones 
ortopédicas o neurológicas que limitaran la aplicación del test. Se contactó telefónicamente a los pacientes en seguimiento cardiológico residentes en la Región Metropolitana de Chile. Se documentaron los datos demográficos y los antecedentes mórbidos de cada paciente mediante una encuesta a padres y/o tutores o mediante la revisión de la ficha clínica electrónica.

Se categorizó el diagnóstico cardiológico inicial según la morfología ventricular en 3 grupos: CUV derecho, CUV izquierdo y ventrículo de morfología indeterminada y/o biventricular. La edad al momento del BPTVD se expresó en meses de vida, y se distinguieron aquellos que fueron intervenidos en forma temprana (menor de 36 meses de vida o igual) versus los intervenidos en forma tardía (mayor de 36 meses de vida). ${ }^{5}$ Según el número de cirugías, los pacientes se clasificaron en dos grupos: aquellos con 3 o menos cirugías cardíacas con circulación extracorpórea (CEC) y aquellos con más de 3 cirugías cardíacas con CEC, y se compararon ambos grupos con los metros alcanzados durante la prueba.

Lo habitual para completar el BPTVD son 2 o 3 etapas quirúrgicas en total. ${ }^{1}$ Un mayor número de cirugías con CEC implica la presencia de defectos residuales y / o complicaciones que afectan en forma negativa la función cardíaca y pulmonar. ${ }^{6}$

El TM6M fue aplicado por dos kinesiólogos según la norma de la American Thoracic Society, ${ }^{7}$ en un espacio cerrado, de superficie plana, de 30 metros de longitud, en cuyos extremos se instalaron 2 conos para señalar el límite del recorrido. Se determinó la distancia total recorrida en metros y se comparó con los valores de referencia en población pediátrica sana. ${ }^{3} \mathrm{Al}$ comenzar y finalizar el TM6M, se registró la frecuencia cardíaca (FC) y la $\mathrm{SatO}_{2}$ con oxímetro de pulso (Nonin Onyx Vantage 9590, Minnesota, USA), presión arterial (PA) medida con esfigmomanómetro digital (GE Critikon Dinamap XL Vital Signs Monitor, USA) y percepción subjetiva de fatiga y disnea utilizando la escala modificada de Borg. ${ }^{8}$ La evaluación nutricional se basó en el índice de masa corporal en relación con la edad (IMC/E) de acuerdo con patrones de la Organización Mundial de la Salud (OMS). ${ }^{9}$

\section{Análisis estadístico}

Para el análisis estadístico, se utilizó el software SPSS V25.0 (NY, USA). Para la descripción de las variables numéricas, se utilizó mediana y rango intercuartil (RIC). Para muestras independientes, se realizó el test de U de Mann-Whitney y, para muestras dependientes, el test de prueba de los rangos con signo de Wilcoxon. Se realizó el test de Kruskal-Wallis y postest de Dunn para evaluar la distancia recorrida en el TM6M con diagnóstico cardiológico inicial o número de cirugías.

Se realizó una regresión lineal múltiple ajustada según edad, peso, talla, IMC, PA sistólica, PA diastólica, PA media, $\mathrm{FC}$ y $\mathrm{SaO}_{2}$ para la distancia recorrida en el TM6M. Se consideró significativo todo valor de $p<0,05$.

\section{Ética}

El estudio fue aprobado por el Comité de Ética en Investigación de la Escuela de Medicina de la Pontificia Universidad Católica de Chile (ID code: 161205006). Los padres y/o tutores firmaron un consentimiento informado, y los pacientes mayores de 7 años, un asentimiento informado.

\section{RESULTADOS \\ Características clínicas y demográficas}

Un total de 105 pacientes con CUV fueron operados de BPTVD entre los años 2000 y 2013, de los cuales 30 pacientes fueron enrolados para el TM6M según el flujograma de la Figura 1. A

FIGURA 1. Flujograma de los pacientes con corazón univentricular en estadio pos-bypass total del ventrículo derecho operados entre el año 2000 y el 2013 en el Hospital Clínico Red Salud UC-Christus

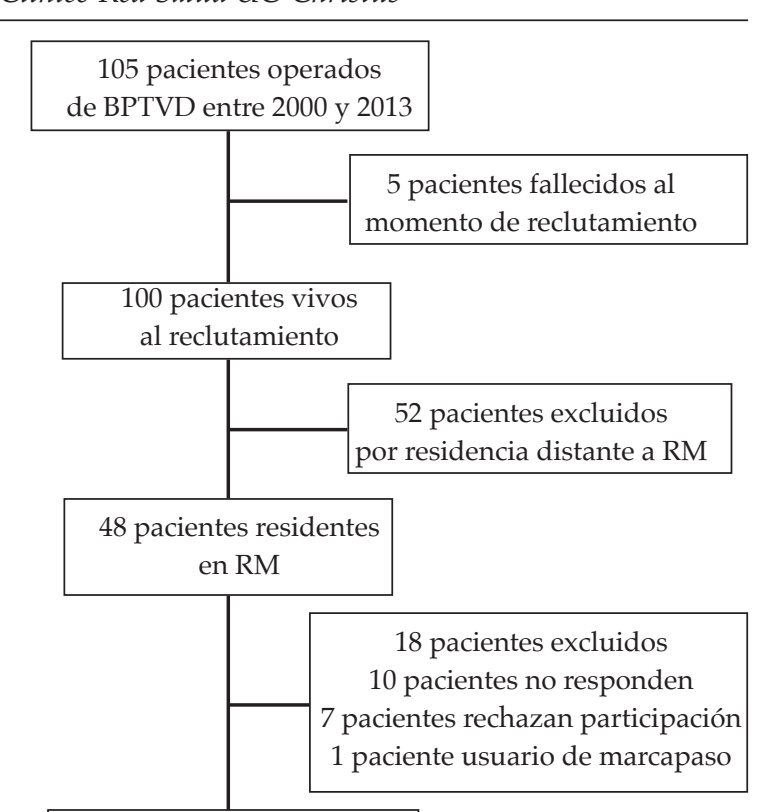

\footnotetext{
30 pacientes evaluados con test de marcha en $6 \mathrm{~min}$
}

BPTVD: bypass total del ventrículo derecho. RM: Región Metropolitana de Chile. 
todos los pacientes incluidos en el estudio se les realizó BPTVD con tubo extracardíaco, de los cuales 26 fueron fenestrados. Las características demográficas y clínicas de los pacientes enrolados se encuentran resumidos en la Tabla 1.

\section{Resultados del test de marcha en $\mathbf{6}$ minutos}

Todos los pacientes completaron el test sin interrupciones. No se observaron complicaciones durante el TM6M ni al término de este. Los parámetros fisiológicos pre- y postest se observan en la Tabla 2. Al aplicar la escala de Borg modificada al finalizar el test, 27 pacientes reportaron algún grado de disnea, y 21, algún grado de fatiga muscular. La mediana de distancia recorrida al finalizar la prueba fue de 551,3 metros, que correspondió a un $84 \%$ de la distancia teórica promedio para la población pediátrica sana, diferencia que fue estadísticamente significativa $(p<0,001)$. Al analizar los factores que pudieran influir con la distancia recorrida, se observó que no existía asociación con el sexo $(p=0,85)$, el estado nutricional $(p=0,52)$, la edad precoz de realización del BPTVD $(p=0,1)$, el diagnóstico cardiológico inicial $(p=0,57)$ ni el número de cirugías con CEC mayor de $3(p=0,37)$. Se realizó un modelo de regresión lineal múltiple como predictor de la distancia recorrida en el TM6M con las variables demográficas y fisiológicas previas a la prueba. Se obtuvo que la talla, la PA sistólica pretest y la $\mathrm{SaO}_{2}$ en reposo fueron significativas $\left(p<0,05 ; R^{2} 0,58\right)$ (Tabla 3$)$.

\section{DISCUSIÓN}

Los pacientes con fisiología de CUV evaluados con el TM6M recorrieron una menor distancia que los pacientes sanos de la misma edad estudiados por Geiger et al. ${ }^{3}$ Esta diferencia en los metros caminados se ha asociado con la patología de base o las diferencias en talla entre ambas poblaciones. ${ }^{4,10,11}$ En cuanto a los posibles factores determinantes de la distancia recorrida, no se encontró relación significativa con sexo, morfología ventricular, número de cirugías previas, estado nutricional y edad al momento del BPTVD. En otras series, estos factores han mostrado influir en la capacidad aeróbica..$^{4-6,12,13}$ Esto podría deberse a que nuestro tamaño muestral fue pequeño y heterogéneo.

TABLA 1. Características demográficas y clínicas de los pacientes reclutados con corazón univentricular en estadio pos-bypass total del ventrículo derecho $(N=30)$

\begin{tabular}{lc}
\hline Característica & Pacientes, $\mathbf{n}(\%)^{\mathbf{a}}$ \\
\hline Sexo masculino & $18(60)$ \\
Edad (años) & $12(8,75-14)$ \\
Talla (cm) & $145(129-156)$ \\
Estado nutricional & \\
$\quad$ Desnutrido & $2(6,7)$ \\
$\quad$ Eutrofia & $18(60)$ \\
$\quad$ Sobrepeso & $9(30)$ \\
$\quad$ Obesidad & $1(3,3)$ \\
Diagnóstico cardiológico & $17(56,7)$ \\
$\quad$ Ventrículo único derecho & $9(30)$ \\
$\quad$ Ventrículo único izquierdo & $4(13,3)$ \\
$\quad$ Biventricular & $21(70)$ \\
No de cirugías & $9(30)$ \\
$\quad 3$ cirugías con CEC & 3 cirugía con CEC \\
Edad al momento del BPTV (meses) & $36,5(33-41)$ \\
Distancia caminada (m) & $551,3(502,3-603,8)$ \\
\hline
\end{tabular}

${ }^{a}$ Datos presentados como n (\%) o como mediana (rango intercuartil)

BPTVD: bypass total del ventrículo derecho.

CEC: circulación extracorpórea.

TABla 2. Parámetros fisiológicos, disnea y fatiga según la escala de Borg modificada pre- y postest de marcha en 6 minutos en pacientes con corazón univentricular en estadio pos-bypass total del ventrículo derecho $(N=30)^{a}$

\begin{tabular}{|c|c|c|c|}
\hline Variable & Pre-TM6M & Pos-TM6M & Valor de $p$ \\
\hline$\overline{\mathrm{FC}}, \mathrm{lpm}$ & $81(58-92)$ & $118,5(103-125)$ & $<0,001$ \\
\hline Saturación de oxígeno, $\%$ & $95(92-96)$ & $92(88-94)$ & $<0,001$ \\
\hline PAS, $\mathrm{mmHg}$ & 109 (104-119) & $113,5(108-127)$ & 0,002 \\
\hline $\mathrm{PAD}, \mathrm{mmHg}$ & $61(55-66)$ & $68(62-73)$ & $<0,001$ \\
\hline Disnea & $0(0-1)$ & $4(3-5)$ & $<0,001$ \\
\hline Fatiga & $0(0-0)$ & $0(0-4)$ & $<0,001$ \\
\hline
\end{tabular}

${ }^{a}$ Datos entregados como mediana con rango intercuartil.

FC: frecuencia cardíaca; lpm: latidos por minuto; PAS: presión arterial sistólica; PAD: presión arterial diastólica; TM6M: test de marcha en 6 minutos. 
TABLA 3. Modelo de regresión lineal múltiple ajustado para la distancia recorrida en el test de marcha en 6 minutos en pacientes reclutados con corazón univentricular en estadio pos-bypass total del ventrículo derecho $(N=30)$

\begin{tabular}{lcc}
\hline Variable & $\beta$ & Valor de $p$ \\
\hline Edad (años) & 0,022 & 0,93 \\
Peso $(\mathrm{kg})$ & $-0,191$ & 0,51 \\
Talla $(\mathrm{m})$ & 0,47 & $0,001^{*}$ \\
$\mathrm{IMC}\left(\mathrm{kg} / \mathrm{m}^{2}\right)$ & $-0,93$ & 0,53 \\
PAS pre-TM6M & 0,29 & $0,043^{*}$ \\
PAD pre-TM6M & $-0,065$ & 0,65 \\
PAM pre-TM6M & 0,017 & 0,94 \\
$\mathrm{FC}$ pre-TM6M & $-0,065$ & 0,64 \\
$\mathrm{SaO}_{2}$ pre-TM6M & 0,384 & $0,006^{*}$ \\
\hline
\end{tabular}

R: 0,$76 ; R^{2}: 0,58$.

* Significativo.

IMC: índice de masa corporal; PAS: presión arterial sistólica; PAD: presión arterial diastólica; PAM: presión arterial media; TM6M: test de marcha en 6 minutos; FC: frecuencia cardíaca; $\mathrm{SaO}_{2}$ : saturación arterial de oxígeno.

Respecto a los parámetros fisiológicos pre- y postest, se observó una respuesta cronotrópica y presora fisiológica con el ejercicio, acorde a lo previamente descrito. ${ }^{14}$ Es importante mencionar que la disminución en la $\mathrm{SatO}_{2}$ postest observada en algunos pacientes podría ser explicada por la presencia de fenestración permeable, conexiones venovenosas o fístulas arteriovenosas. ${ }^{14,15} \mathrm{E} 1$ modelo de regresión lineal múltiple determinó una ecuación en la que la talla, la PA sistólica y la $\mathrm{SatO}_{2}$ pretest predecían, en un $58 \%$, la distancia caminada en nuestra muestra de pacientes.

Si bien la prueba de ejercicio cardiopulmonar (PECP) es la más objetiva en la medición de la $\mathrm{CF},{ }^{14}$ el TM6M tiene dos ventajas descritas sobre el primero: su simplicidad y bajo costo, y que, al ser un test de ejercicio submáximo, puede ser realizado por aquellos que no toleran el ejercicio máximo. ${ }^{7}$ Además, su similitud con actividades de la vida diaria posicionan el TM6M como una prueba que logra determinar la CF de estos pacientes, con una buena correlación entre la distancia caminada y el consumo de oxígeno medido por la PECP. ${ }^{4}$

Dentro de las limitaciones de este estudio se encuentra el tamaño muestral pequeño y no estratificado, además de una muestra no aleatoria con selección por conveniencia. Se necesitan más estudios que consideren una muestra estratificada por grupo etario y sexo para lograr mayor representatividad de esta población de pacientes que nos permitan obtener valores de referencia para realizar estudios comparativos y, así, poder sentar las bases para el desarrollo de programas de rehabilitación cardíaca pediátrica.

\section{REFERENCIAS}

1. Rychik J, Atz A, Celermajer D, Deal B, et al. Evaluation and managment of the child and adult with fontan circulation. A Scientific Statement from the American Heart Association. Circulation. 2019; 140:e234-84.

2. Ohuchi H, Negishi J, Noritake K, Hayama Y, et al. Prognostic value of exercise variables in 335 patients after the Fontan operation: a 23-year single-center experience of cardiopulmonary exercise testing. Congenit Heart Dis. 2015; 10(2):105-16.

3. Geiger R, Strasak A, Treml B, Gasser K, et al. Six-minute walk test in children and adolescents. J Pediatr. 2007; 150(4):395-9.e1-2.

4. Moalla W, Gauthier R, Maingourd Y, Ahmaidi S. Sixminute walking test to assess exercise tolerance and cardiorespiratory responses during training program in children with congenital heart disease. Int J Sports Med. 2005; 26(9):756-62.

5. Daley M, d'Udekem Y. In patients undergoing Fontan completion, does a younger age at operation result in better long-term exercise capacity and prognosis? Interact Cardiovasc Thoracic Surg. 2019; 28(2):301-5.

6. Jacobs JP, Mavroudis C, Quintessenza JA, Chai PJ, et al. Reoperations for pediatric and congenital heart disease: an analysis of the Society of Thoracic Surgeons (STS) congenital heart surgery database. Semin Thorac Cardiovasc Surg Pediatr Card Surg Annu. 2014; 17(1):2-8.

7. ATS Committee on Proficiency Standards for Clinical Pulmonary Function Laboratories. ATS statement: guidelines for the six-minute walk test. Am J Respir Crit Care Med. 2002; 166(1):111-7.

8. Borg GA. Psychophysical bases of perceived exertion. Med Sci Sports Exerc. 1982; 14(5):377-81.

9. Word Health Organization. WHO reference 2007. Growth reference data for 5-19 years. [Acceso: 14 de mayo de 2020]. Disponible en: https: / / www.who.int/growthref/en/.

10. Feltez G, Coronel CC, Pellanda LC, Lukrafka JL. Exercise capacity in children and adolescents with corrected congenital heart disease. Pediatr Cardiol. 2015; 36(5):1075-82.

11. Schaan CW, Feltez G, Schaan BD, Pellanda LC. Functional capacity in children and adolescents with congenital heart disease. Rev Paul Pediatr. 2019; 37(1):65-72. 
12. Kamata M, Stiver C, Naguib A, Tumin D, et al. A retrospective analysis of the influence of ventricular morphology on the perioperative outcomes after fontan surger. J Cardiothorac Vasc Anesth. 2017; 31(1):128-33.

13. Makni E, Elloumi A, Ben Brahim M, Moalla W, et al. Sixminute walk distance equation in children and adolescents with obesity. Acta Paediatr. 2020 [En prensa].
14. Rhodes J. Fontan circulation. In Rhodes J, Alexander M, Opotowsky A (eds.). Exercise Physiology for the Pediatric and Congenital Cardiologist. Switzerland: Springer; 2019. Págs.97-115.

15. Sen S, Bandyopadhyay B, Eriksson P, Chattopadhyay A. Functional capacity following univentricular repair-midterm outcome. Congenit Heart Dis. 2012; 7(5):423-32.

\section{Artículos seleccionados}

Los siguientes resúmenes y comentarios de trabajos seleccionados se encuentran disponibles en la versión electrónica de este número.

ARCH DIS CHILD FETAL NEONATAL ED, 2020; 0:F1-F9.

Escurrimiento del cordón umbilical en niños prematuros: revisión sistemática y meta-análisis (Balasubramanian $\mathrm{H}$, et al. Umbilical cord milking in preterm infants: a systematic review and meta-analysis).

Comentario: Dr. José María Ceriani Cernadas. Editor Archivos Argentinos de Pediatría.

PEDIATRICS. 2020 Apr;145(4):e20200204.

Recursos recomendados para la atención de los pacientes pediátricos en los hospitales (Ernst KD. Resources recommended for the care of pediatric patients in hospitals).

Comentario: Dra. Paula Otero. Hospital Italiano de Buenos Aires.

ARCH DIS CHILD. 2020;archdischild-2020-319833. [published online ahead of print, 2020 Jun 18].

Impacto de la pandemia por COVID-19 en las consultas al departamento de emergencias pediátricas en Argentina (Ferrero F, et al. Impact of the COVID-19 pandemic in the paediatric emergency department attendances in Argentina).

Comentario: Dra. M. Susana Rodríguez. Hospital de Pediatría Prof. Dr. J. P. Garrahan. Ciudad de Buenos Aires.

PEDIATRICS. 2020 Jul;146(1):e20193518.

Uso de celulares inteligentes y tablets en niños pequeños (Radesky JS, et al. Young children's use of smartphones and tablets).

Comentario: Dr. Fernando Lamas. Subcomisión Tecnologías de Información y Comunicación SAP.

PEDIATRICS. 2020 Jun;145(6):e20193621.

Leyes sobre manejo con distracción y accidentes fatales de vehículos automotores (Flaherty MR, et al. Distracted driving laws and motor vehicle crash fatalities).

Comentario: Dr. Lucas David Navarro. Hospital de Niños Dr. Orlando Alassia. Santa Fe.

PEDIATRICS. 2020;145(6):e20193728.

Biomarcadores y gravedad de la enfermedad en niños con neumonía adquirida en la comunidad (Florin TA, et al. Biomarkers and disease severity in children with community-acquired pneumonia).

Comentario: Dres. Gabriela Sanaluis Fenelli y Fernando Torres. Comité de Docencia e Investigación. Hospital General de Niños Pedro de Elizalde. Ciudad de Buenos Aires. 